\title{
Direct gene transfer for immunotherapy and immunization
}

The immune response to infectious organisms is dependent on the recognition of the products of 'foreign' genes expressed within host cells. The concept of immunization therefore arises as a natural consequence of gene transfer in mammals, and using recombinant molecular-genetic approaches to modulate immune function represents a promising avenue of investigation for the prevention and treatment of cancer and infectious diseases. Immune recognition depends on the effective synthesis of foreign gene products, together with appropriate presentation of these proteins to the immune system by the major histocompatibility complex (MHC).

During the past few decades, the molecular and genetic bases of immune recognition have been elucidated. The roles of the histocompatibility complex glycoproteins and the antigen-recognition structures, including antibody molecules and the $\mathrm{T}$-cell receptor, have been defined at the molecular level. Through such studies, it has become evident that these immunological recognition structures have evolved to identify foreign proteins that are synthesized within cells, and to present them in a manner that pcrmits immunological recognition and response.

In addition to these advances in understanding the genetic basis of immune recognition, considerable progress has been made in developing recombinant gene-transfer techniques in vivo. In nature, transfer of foreign genetic material by viruses and microorganisms often elicits effective immune responses, and successful vaccinations based on the deliberate introduction of foreign vectors are well-documented.

Attenuated virus vaccines, beginning with the use of the cowpox virus by Jenner to protect against smallpox infection, Pasteur's rabies vaccine, and numerous subsequent attenuated virus vaccines including polio, rubella, measles, mumps and yellow fever, represent widely tested and effective treatments in modern medicine. These approaches to immunization involve direct in vivo delivery and expression of genes, i.e. genetic immunization in its most primitive form. In addition, these studies have documented the efficacy and potential therapeutic benefits that can be derived

G. J. Nabel is at the Howard Hughes Medical Institute, University of Michigan Medical Center, 1150 W. Medical Center Drive, 4510 MSRB I, Ann Arbor, MI 48109-0650, USA. P. Felgner is at Vical Inc., 9373 Toune Centre Drive, Suite 100, San Diego, CA 92121, USA. when foreign genetic material is introduced directly into humans in vivo to prevent infection by specific pathogens.

With the advent of molecular genetics and the more-recent advances in gene-transfer techniques, it has become apparent that gene-transfer approaches can now be used efficiently and safely to address clinical problems of major medical significance. In particular, gene-transfer methods can be used to stimulate the immune system to recognize viruses, parasite-infected cells and cancerous cells. Such approaches could potentially be used to address major threats to public health, such as those posed by acquired immunodeficiency syndrome (AIDS), tuberculosis and Lyme disease. In this review, the potential application of direct gene transfer to immunotherapy and immunization for treating diseases such as viral infections and cancer is discussed.

\section{Emergence of gene-delivery technology}

Efforts to identify methods for enhancing the delivery of functional polynucleotides into living cells were stimulated in the mid 1950 s by the results of Alexander et al. (reviewed in Ref. 1), which demonstrated that purified poliovirus RNA was infectious in HeLa cells. Although the infectivity of the purified genomic RNA was extremely low when compared with the original intact virus (up to one million times less active), this nevertheless showed that living virus particles were not required to produce competent, infectious virus in vivo. Moreover, the investigators showed that a hypertonic saline solution $(1 \mathrm{M} \mathrm{NaCl})$ could enhance infectivity of the purified RNA by $\sim 100$-fold. These humble beginnings led to improved methods for cellular transfection, including calcium phosphate co-precipitation ${ }^{2}$ and diethyl aminoethyl (DEAE)-dextran ${ }^{3}$. Subsequent experiments using the calcium-phosphate procedure demonstrated that transfection of a non-infectious fragment of the herpes simplex virus (HSV) genome containing the thymidine kinase (TK) gene could transform $\mathrm{TK}^{-}$ cells ${ }^{4}$, i.e. an infectious and proliferating virus was unnecessary for cellular transformation. Transfection technology converged with the emerging recombinant DNA technology in the late 1970 s when Berg and colleagues used calcium-phosphate, DEAEdextran and liposomal transfection methods to deliver and express recombinant plasmids in cultured mammalian cells ${ }^{5,6}$. 


\section{targeting specific diseases}

\section{Effective non-viral delivery methods}

The efficiency with which intact viruses introduce genes into cultured cells led to the search to identify synthetic systems that could emulate viral delivery while avoiding viral infectivity ${ }^{1,6,7}$. The two intrinsic viral attributes that have attracted the most attention in this regard are the specific interaction with cellsurface receptors on target cells, and viral membranefusion proteins. Receptors enable infectious particles to attach specifically to the surface of target cells, whereas fusion proteins enable delivery of the viral genome into the cytoplasm of the host cell following receptor-mediated endocytosis. Viruses lacking a membrane-fusion capability would be trapped in the lysosomal pathway of the cell and would be degraded by hydrolytic and oxidizing enzymes located in the lysosomes. In principle, fusion events leading to functional polynucleotide delivery can occur within the phagocytic vesicle compartment (phagosome), within the lysosomal compartment, or directly with the cellsurface plasma membrane. However, two major technical hurdles have prevented the widespread application of conventional liposomes as vehicles for gene delivery. (1) The efficiency of DNA encapsulation into conventional liposomes is usually very low because the internal volume of the vesicle is too small to encapsulate macromolecules the size of genes efficiently and conveniently. (2) The transfection efficiency of liposomal DNA is also very low because the efficiency with which liposomes bypass the lysosomal pathway is still too low. Liposomes that contain receptors that are capable of interacting with specific target cells have been prepared and tested; they are capable of bypassing the lysosomal compartment by a protein-mediated or $\mathrm{pH}$-sensitive fusion mechanism. Two additional gene-transfer approaches which do not use viral vectors, and which can also overcome some of the problems encountered with conventional liposomes, are direct intramuscular injection of genetic material into tissue, and in vivo lipofection using cationic lipid-DNA complexes.

\section{Polycationic delivery systems}

The commercially available cationic-lipid vector, Lipofectin $^{8,9}$, is one member of a class of cationic-lipid formulations called 'cytofectins' that can overcome many of the problems associated with the use of conventional liposomes as gene-delivery vehicles. Cytofectin formulations consist of cationic liposomes which have three properties that are of particular importance to the delivery of polynucleotides.

- These liposomes condense with DNA to form complexes in which all the DNA is entrapped, regardless of its size.

- Virtually all biological surfaces, including cell surfaces, carry a net negative charge. Thus, positivelycharged lipid vesicles containing gene sequences interact spontaneously with cell surfaces, delivering the associated polynucleotide to the cell.

- Cationic lipid-polynucleotide complexes fuse with cell membranes in a manner that allows the entrapped DNA to avoid degradation in the lysosomal compartment.

Although receptor-mediated phagocytosis usually enables even conventional liposomes to enter cells in reasonable amounts, these particles do not easily escape the lysosomal compartment of the cell, which completely degrades the liposomes and the encapsulated DNA. The cytofectin-mediated fusion, which leads to escape from lysosomal degradation, depends on the structure of the cationic lipid molecule, as well as on the presence of neutral lipids in the final formulation.

Another notable polynucleotide-delivery system involving the use of polycations is the polylysineligand conjugate system of $\mathrm{Wu}^{10}$ and Birnstiel ${ }^{11}$. This system employs specific ligands, conjugated to polylysine, that spontaneously condense with DNA through disassociable ionic interactions. The resulting condensate is taken up by receptor-mediated endocytosis into cells containing the receptor for the conjugated ligand. The addition of chloroquine or adenovirus coat proteins can be used to disrupt the lysosomal compartment and release DNA into the cell.

\section{Exploiting viral sequences for in vivo gene delivery}

The use of viruses as gene-transfer vehicles arose from the observation that viruses are extremely efficient at introducing genes into their host cells, and that they might therefore be useful as vectors for introducing genes to correct genetic diseases in man. In particular, since DNA and RNA tumor viruses integrate into mammalian genomes, thus propagating the introduced genes on cell division, it was proposed that, if the deleterious features of these viruses could be eliminated, they might be able to deliver safely a desirable gene to correct the cellular defects of genetic disorders $^{12,13}$. Substantial progress has been made, in the past decade, towards the production of safe and effective retroviral vectors but, until recently, injecting such vectors has been considered only for use in ex vivo protocols, not for direct in vivo gene transfer.

Subsequently, it was found that it might be possible to exploit features of viral sequences in improving infectivity of introduced plasmid DNA, without the need for including infectious viruses. Among the earliest reported direct in vivo transfections using cloned genes without the use of infectious virus vectors were experiments showing that virus replication could occur following injection of cloned infectious viral plasmid DNA in vivo (reviewed in Ref. 14). The plasmid DNA used for these studies was purified from bacteria and therefore did not contain any viral particles or proteins to facilitate uptake into the cell. These experiments contributed to a better understanding of the role of specific viral genes in in vivo virus replication and oncogenesis, although at the time, little attention was paid to the possibility of using the apparent low level of expression for therapeutic benefit. 


\section{targeting specific diseases}

\section{Direct in vivo, non-viral gene delivery}

Several different approaches have been used to introduce and obtain expression from non-replicating plasmid sequences in vivo. In addition to the use of techniques (e.g. liposomes, calcium phosphate precipitates and polylysine conjugates) mentioned earlier, successful gene expression following direct injection of 'naked' nucleic acids and cationic-lipid-mediated delivery have been reported.

\section{Direct injection}

In a systematic survey to identify tissues permissive for in vivo delivery, we found that, in skeletal muscle, no cationic-lipid vector was necessary to achieve significant levels of transgene expression ${ }^{15}$. Up to one nanogram of reporter-gene product [luciferase, chloramphenicol acetyltransferase (CAT) or $\beta$-galactosidase] could be isolated from skeletal muscle injected in vivo with 10-100 mg of plasmid DNA. Although there was no evidence of integration into the host genome, expression persisted for more than six months. (Integration was not expected in the fully differentiated non-dividing muscle fibers in which the expression was detected, since integration requires cell division.) Persistent expression implied that the introduced sequences were maintained and repaired in the nucleus like genomic DNA. In vitro transcribed mRNA was also taken up and expressed in mousc muscle, but the duration of expression was much shorter. Several reports have subsequently demonstrated similar results with direct gene delivery in rat cardiac muscle ${ }^{16}$. Directly injected recombinant DNA was expressed by a small percentage of cardiac myocytes in the area of injection, and the genes introduced in this way are regulated normally by hormonal signals. Another form of direct gene delivery, other than direct injection, that has been used to introduce functional genes into living mammalian tissue is high energy microparticle bombardment ${ }^{17}$. This approach has been used successfully to generate immune responses in vivo (see below)

\section{Cationic lipid-DNA complexes}

A number of studies have shown that cationiclipid-mediated reporter-gene transfer can mediate effective gene delivery in vivo, either by injection (into neural or lung tissue) ${ }^{14}$, or by direct administration into the lung by aerosol or intubation ${ }^{18-20}$.

A catheter-based gene-delivery system has now also been used to localize the expression of recombinant genes to the anatomical site of introduction. Plasmids can be transferred by lipofection into the arterial wall using a double-balloon catheter which isolates a space between the two balloons. Expression of different recombinant genes has been documented $\mathrm{d}^{21-25}$ in porcine endothelial, vascular smooth muscle, and adventitial cells. Similar results have been reported in other species ${ }^{26,27}$. The arterial or venous circulation can also be used as a conduit to introduce genes into organs, or to sites of focal pathology, for example, in tumor nodules (see below).

\section{Direct gene transfer and modulation of the immune system \\ Immunotherapy}

Early studies used catheter-based gene delivery as a model system to demonstrate that specific reporter genes could be expressed in vivo ${ }^{21,22}$. Subsequent studies investigated whether specific biological responses (i.e. immune responses) could be induced at sites of recombinant gene transfer. A highly immunogenic foreign MHC molecule was used to elicit an immune response in the pig iliofemoral artery. Using direct gene transfer (either a retroviral vector or DNA-liposome complex ${ }^{24}$ ), the human HLA-B7 gene was introduced into the artery and its expression was detected at specific sites within the vessel wall. More importantly, expression of this foreign MHC antigen induced an immunological response at the sites of genetic modification. (The response included a granulomatous mononuclear cell infiltrate beginning ten days after introduction of the recombinant gene. This response resolved by 75 days after gene transfer; however, a specific cytolytic $\mathrm{T}$-cell response against the HLA-B7 molecule was persistent.) Not only was a specific immunological response induced by the introduction of a recombinant gene at a specific site in vivo, but also this study showed that direct gene transfer of specific recombinant genes could elicit an immune response to the product of that gene in vivo 24 . In addition, this approach provided a general method for the induction of an immune response at a specific site in vivo.

To determine whether direct gene transfer might be appropriate for the treatment of disease, a murine model of malignancy has been developed. Direct gene transfer of an allogeneic (i.e. of different genetic origin) MHC gene into a murine tumor elicits a T-celldependent immune response, not only to the foreign MHC gene, but also to previously unrecognized tumor-associated antigens, which are now recognized within the context of the 'self' MHC. Thus, the immune system acquires the ability to rccognize and lyse tumor cells which had previously cvaded detection.

In animals presensitized to a specific MHC haplotype, direct gene transfer into established tumors could attenuate tumor growth or, in some cases, lead to complete tumor regression ${ }^{28}$. Direct gene transfer of foreign MHC genes into tumors thus has potential therapeutic effects that may be appropriate for the treatment of malignancy.

As an extension of this work, a clinical trial based on direct gene transfer received approval of the Recombinant DNA Advisory Committee (RAC) of the National Institutes of Health (NIH) on 10 January, 1992. This clinical trial involved the treatment of subcutaneous lesions of patients with metastatic melanoma. The treatment consists of intratumoral injection of the human class I MHC gene, HLA-B7, complexed to a cationic liposome Dope/ $3 \beta$ [N', N'-dimethylaminoethane-carbamoyl] cholesterol $^{29,30}$ in escalating doses. Toxicity, expression of 


\section{targeting specific diseases}

the recombinant gene, and the immunological response to treatment are currently being evaluated. The objectives of this first human clinical trial using direct gene transfer are: (1) to establish a safe and effective dose of DNA-liposome complex to introduce recombinant genes in vivo; (2) to confirm expression of the transduced gene; and (3) to analyse the immune response and potential therapeutic effects of this method of therapy. Toxicity has not readily been apparent in both long-term and short-term animal studies using these modes of direct gene transfer in vivo $29,31,32$. Taken together, these studies will determine whether direct gene transfer is an appropriate form of treatment for malignancy, and whether this method of delivery might also be appropriate for treating other human diseases.

\section{Immunization}

Two different pathways of antigen processing lead to antigen presentation: either through $\mathrm{MHC}$ class I or $\mathrm{MHC}$ class II molecules ${ }^{33}$. Extracellular antigens are taken up endocytically and the protease-digested fragments are presented by the MHC class II molecules. This stimulates CD4+ helper $\mathrm{T}$ cells and amplifies the humoral immune response. Intracellular antigen synthesis leads to presentation by MHC class I molecules and stimulation of $\mathrm{CD}^{+}$cytotoxic $\mathrm{T}$ cells (CTLs). The ability to introduce foreign genes which can be expressed (i.e. intracellular antigen synthesis) enables the MHC class I pathway to be preferentially stimulated and specific $\mathrm{CD}^{+}$cytotoxic $\mathrm{T}$-cell responses to be increased.

Humoral responses are involved in the neutralization of active pathogens, whercas cellular CTL responses eliminate infected cells that are the source of the pathogens. The live, attenuated virus vaccines in current use activate both the cellular and humoral arms of the immune system, yielding a balanced response; this class of vaccines is generally more effective, more cross-reactive and gives effects of a longer duration than the responses induced by inactivated vaccines. The cross-reactivity of the vaccine is particularly important for viruses such as influenza and human immunodeficiency virus (HIV) that undergo progressive antigenic drift.

We undertook studies to determine whether direct administration of plasmids encoding viral antigens that are subsequently expressed in muscle tissue could produce a balanced immune response, similar to that associated with attenuated virus vaccines ${ }^{34}$. Balb/c mice were treated with a single $50 \mu \mathrm{l}$ intramuscular injection of $100 \mu \mathrm{g}$ of a plasmid containing the gene for a secreted form of the HIV gp120 protein and driven by the CMV immediate-early promoter. High titer IgG antibodies directed against the gp 120 protein were produced and maintained for $>6$ months. The same injection procedure also induced potent, longlasting and specific CTL responses against the gp120 CTL peptide epitope.

To assess more thoroughly the quality of the immune responses generated following injection of plasmids encoding viral antigens, we used the mouse influenza model to carry out antiviral efficacy studies ${ }^{35,36}$. Plasmid cDNA encoding nucleoprotein (from the Puerto Rico strain of influenza virus) injected into the quadriceps muscle of $\mathrm{Balb} / \mathrm{c}$ mice produced potent antibody and CTL responses against nucleoprotein. The results of the quantitative immunological assays demonstrated that a single injection of nucleoprotein DNA generated the appropriate nucleoprotein-derived peptide in association with $\mathrm{MHC}$ class I antigen, for induction of the specific CTL response. These CTLs could recognize the nucleoprotein peptide epitope generated on naturally infected cells.

The in vivo protective efficacy of the nucleoprotein-DNA immunization was demonstrated in several ways. Following challenge with live influenza virus, the nucleoprotein-DNA vaccinated mice had much lower influenza virus titers in their lungs, less weight loss due to infection, and greater survival from a lethal virus challenge than control-vaccinated animals. Thus, the use of an expression vector encoding a viral protein as an immunogen offers a simple means to induce cross-strain protective immunity, without the need for live attenuated virus adjuvants or viral genetransfer vectors.

\section{Future directions}

Judging from the magnitude and quality of data generated during the last three years, the prospects for direct in vivo gene delivery are very promising. Cytofectin formulations have been shown to facilitate delivery and gene expression in skin, brain and liver via direct injection into the tissue. In addition, other vectors, including polylysine--ligand complexes and viruses, have yielded expression following gene transfer in vivo. Expression of specific recombinant genes in these tissues provides a potent method to induce immune reactivity against a variety of gene products. After direct intramuscular injection, recombinant viral genes have induced immune responses which provide protective immunity against live virus infections, paving the way towards a new class of more effective vaccines. Finally, direct gene transfer has been successful in providing immunotherapcutic responses in murine models of malignancy. Based on these studies, a clinical protocol has been initiated to analyse the potential toxicity and efficacy of this mode of treatment in humans. If this form of treatment is welltolerated, direct gene transfer could simplify and facilitate molecular-genetic interventions for a variety of human diseases.

\section{References}

1 Felgner, P. L. (1990) Adv. Drig Deliv. Rev. 5, 163-187

2 Graham, F. L. and van der Eb, A. J. (1973) Virology 52, 456-467

3 Vaheri, A. and Pagano, J. S. (1965) Virology 27, 434-436

4 Minston, A. C., Wildy, P., Buchan, A. and Darby, G. (1978) Cell 13, $481-487$

5 Lee, F., Mulligan, R., Berg, P. and Ringold, G. (1981) Nature 294, 228-232

6 Fraley, R., Subramani, S., Berg, P. and Papahadjopoulos, D. (1980) 


\section{targeting specific diseases}

J. Biol. Chem. 255, 10431-10435

7 Loyter, A., Vainstein, A., Graessmann, M. and Graessmann, A. (1983) Exp. Cell Res. 143, 415-425

8 Felgner, P. L., Gadek, T. R., Holm, M., Roman, R., Chan, H. W., Wenz, M., Northrop, J. P., Ringold, G. M. and Danielsen, M. (1987) Proc. Natl Acad. Sci. USA 84, 7413-7417

9 Felgner, P. L. and Ringold, G. M. (1989) Nature 337, 387-388

10 Wu, C. H., Wilson, J. M. and Wu, G. Y. (1987) J. Biol. Chem. 264, 16985-16987

11 Cotten, M., Wagner, E., Zatloukal, K., Phillips, S., Curiel, D. T. and Birnstiel, M. L. (1992) Proc. Natl Acad. Sci. USA 89, 6094-6098

12 Andersen, F. W. (1984) Science 226, 401-409

13 Friedman, T. (1989) Science 244, 1275-1281

14 Felgner, P. L. and Rhodes, G. H. (1991) Nature 349, 351-352

15 Wolff, J. A., Malone, R. W., Williams, P., Chong, W., Acsadi, G., Jani, G. and Felgner, P. L. (1990) Science 247, 1465-1468

16 Leinwand, L. A. and Leiden, J. M. (1991) Trends Cardiovasc. Med. 1, $271-276$

17 Johnston, S. A. (1990) Nature 346, 776-777

18 Hazinski, T. A., Ladd, P. A. and DeMatteo, C. A. (1991) Am.J. Respir. Cell Biol. 4, 206-209

19 Yoshimura, K., Rosenfeld, M. A., Nakamura, H., Scherer, E. M. Pavirani, A., Lecocq, J-P. and Crystal, R. G. (1992) Nucleic Acids Res. 20, 3233-3240

20 Stribling, R., Brunctte, E., Liggitt, 1)., Gaensler, K. and Debs, R. (1992) Proc. Natl Acad. Sci. USA 89, 11277-11281

21 Nabel, E. G., Plautz, G. and Nabel, G. J. (1990) Science 249, 1285-1288

22 Nabel, E. G., Plautz, G., Boyce, F. M., Stanley, J. C. and Nabel, G. J. (1989) Srience 244, 1342-1344

23 Plautz, G., Nabel, E. G. and Nabel, G. J. (1991) Circulation 83,
578-583

24 Nabel, E. G., Plautz, G. and Nabel, G. J. (1992) Proc. Natl Acad. Soi. USA 89, 5157-5161

25 Lynch, C. M., Clowes, M. M., Osborne, W. R. A., Clowes, A. W. and Miller, A. D. (1992) Proc. Natl Acad. Sci.USA 89, 1138-1142

26 Leclerc, G., Gal, D., Takeshita, S., Nikol, S., Weir, L. and Isner, J. M. (1992) J. Clin. Invest. 90, 936-944

27 Lim, C. S., Chapman, G. D., Gammon, R. S., Muhlestein, J. B., Bauman, R. P., Stack, R. S. and Swain, J. L. (1991) Circulation 83, 2007-2011

28 Plautz, G. E., Yang, Z., Wu, B., Gao, X., Huang, L. and Nabel, G. J. (1993) Proc. Natl Acad. Sci. USA 90, 4645-4649

29 Nabel, G. J. (1992) Hum. Gene Ther. 3, 705-711

30 Gao, X. and Huang, L. (1991) Biochem. Biophys. Res. Commun. 179 , $280-285$

31 Stewart, M. J., Plautz, G. E., Del Buono, L., Yang, Z. Y., Xu, L., Gao, X., Huang, L., Nabel, E. G. and Nabel, G. J. (1992) Hum. Gene Ther. 3, 267-275

32 Nabel, G. J. (1992) Hum.Gene Ther. 3, 399-410

33 Germain, R. N. (1991) Nature 353, 605

34 Rhodes, G. H., Abai, A. M., Dwarki, V. J., Parker, S. E., Manthorpe, M., Felgner, J., Felgner, P. L. and Gromkowski, S. H. Vaccines (in press)

35 Liu, M. A., Ulmer, J. B., Friedman, A., Martinez, D., Hawe, L. A., DeWitt, C. M., Leander, K. R., Shi, X-P., Montgomery, D. L., Donnelly, J. J., Parker, S., Felgner, P. L. and Felgner, J. Vaccines (in press)

36 Ulmer, J. B., Donnelly, J. J., Parker, S. E., Rhodes, G. H., Felgner, P. L., Dwarki, V. J., Gromkowski, S. H., Deck, R. R., DeWitt, C. M., Friedman, A., Hawe, L. A., Leander, K. R., Marinez, D. Perry, H. C., Shiver, J. W., Montgomery, D. L. and Liu, M. A. (1993) Science 259, 1745-1749

\section{TIBTECH Editorial Policy}

Trends in Biotechnology is a news, reviews and commentary journal designed to keep its international readership up-to-date with the current developments in biotechnology. TIBTECH occupies a niche between primary research journals and conventional review journals - it is not a vehicle for the publication of original research data or methods.

There is a strong emphasis on an integrated approach to communicating significant new advances in biotechnology, and discussing their commercial potential. This necessitates combining essential background with state-of-the-art information to make the topics addressed accessible and valuable to newcomers and experts in the field alike. All articles are subject to peer-review and are prepared to strict standards to ensure clarity, scientific accuracy and readability. Commissioning does not guarantee publication.

Reviews: Balanced and concise presentations of specific areas of research. The scope of such reviews is more limited than those of conventional review journals, but provides sufficient information to place the latest developments in a particular field in perspective.

Focus: Short minireview-style articles, which focus on a detailed examination of a narrow topic, thus providing a quick publication response to recognition of significant new directions of research.

Forum: A platform for debate and analytical discussion of newly reported advances - whether reported in the research literature or at conferences. Includes subsections. Workshop, Meeting Reports, and Letters to the Editor.

Biotopics: A column providing a lighter, more personal treatment of topics ranging from serious science and novel techniques, to finance and ethical questions.

Features: Review-length articles which discuss business issues relevant to all biotechnologists patenting, commercial opportunities and regulatory aspects. 